\title{
METHOD OF GENERALIZED FUNCTIONS IN PLANE BOUNDARY VALUE PROBLEMS OF UNCOUPLED THERMOELASTODYNAMICS
}

\author{
Assiyat Dadayeva ${ }^{1}$ and Lyudmila Alexeyeva ${ }^{1}$ \\ ${ }^{1}$ RSE Institute of Mathematics and Mathematical Modeling
}

July 13, 2021

\begin{abstract}
Nonstationary boundary value problems of uncoupled thermoelasticity are considered. A method of boundary integral equations in the initial space-time has been developed for solving boundary value problems of thermoelasticity by plane deformation. According to generalized functions method the generalized solutions of boundary value problems are constructed and their regular integral representations are obtained. These solutions allow, using known boundary values and initial conditions (displacements, temperature, stresses and heat flux), to determine the thermally stressed state of the medium under the influence of various forces and thermal loads. Resolving singular boundary integral equations are constructed to determine the unknown boundary functions.
\end{abstract}

MSQ 74H05; 35K05; 35L05; 35M10

METHOD OF GENERALIZED FUNCTIONS IN

PLANE BOUNDARY VALUE PROBLEMS OF

UNCOUPLED THERMOELASTODYNAMICS

${ }^{1}$ Alexeyeva L.A., ${ }^{2}$ Dadayeva A.N.

${ }^{1}$ Institute of Mathematics and Mathematical Modeling

of Education and Science Ministry of Republic of Kazakhstan,

Almaty, Kazakhstan,alexeeva@math.kz

${ }^{2}$ Institute of Cybernetics and Information Technologies, Satbayev University

Almaty, Kazakhstan,dady1262@mail.ru

https://orcid.org/0000-0002-7131-4635 (Alexeyeva L.A.)

0000-0001-8954-6007 (Dadayeva A.N.)

\section{METHOD OF GENERALIZED FUNCTIONS IN}

PLANE BOUNDARY VALUE PROBLEMS

UNCOUPLED THERMOELASTODYNAMICS

Abstract: Nonstationary boundary value problems of uncoupled thermoelasticity are considered. A method of boundary integral equations in the initial space-time has been developed for solving boundary value problems of thermoelasticity by plane deformation. 
According to generalized functions method the generalized solutions of boundary value problems are constructed and their regular integral representations are obtained. These solutions allow, using known boundary values and initial conditions (displacements, temperature, stresses and heat flux), to determine the thermally stressed state of the medium under the influence of various forces and thermal loads. Resolving singular boundary integral equations are constructed to determine the unknown boundary functions.

Key words: uncoupled thermoelasticity, fundamental solutions, displacements, temperature, stresses, heat flux.

Introduction. The development of thermoelasticity research is associated with the need to develop new mechanical structures, the elements of which operate under conditions of uneven and unsteady heating (in aviation and rocket technology, in nuclear reactor protection systems, in a number of machine-building complexes, industry, etc.). This leads to the appearance of temperature gradients in the medium and deterioration of the strength properties of materials. Thermal shock causes some materials to become brittle and degrade.

In 1956, the work of M. Biot [1] was published, in which a complete substantiation of basic relations and equations of coupled thermoelasticity, based on the laws of thermodynamics of irreversible processes, was given for the first time. This author also formulated the basic variational principles and developed some methods for solving the thermoelasticity equations. In the ensuing publications of V. Novatskiy [2,3], various methods of solving the differential equations of thermoelasticity are proposed, and the models of coupled and uncoupled thermoelasticity are substantiated. According to methods of complete and incomplete separation of variables, he constructed and investigated a number of solutions of these equations and considered a whole class of quasi-static and dynamic problems of thermoelasticity.

In works devoted to dynamic problems of thermoelasticity, the thermal shock problems stand out separately. In formulating such a problem, it is assumed that at the initial moment the object is at rest, and in the subsequent moment there is a sharp change in the thermoelastic state due to the action of heat and power sources, both external and in the medium itself.

Thus, the problem of the propagation of a thermoelastic wave in a half-space due to the instantaneous heating of its boundary for the case of small times was first considered by V.I. Danilovskaya [4] and solved by the small parameters method. A large review of works on thermoelasticity is given by Hetnarski R. in the encyclopedia [5].

In $[6,7]$, the method boundary integral equations (BIE) was developed to solve boundary value problems of coupled and uncoupled thermoelastic elastodynamics. When solving these problems, BIE were constructed in the space of Laplace transforms in time. One of the main problems of the method BIE in the Laplace transform space, which is well known, is the instability of the numerical procedures for inverting transformants of solutions with increasing time, which does not allow constructing solutions in calculations at even small times.

In order to avoid these problems, the method BIE in the initial space-time is being developed here to solve boundary value problems (BVP) of thermoelasticity under plane deformation.

1. Mane relations of thermoelasiticity. An isotropic thermoelastic medium is characterized by a finite number of thermodynamic parameters: mass density $\rho$, elastic Lamé constants $\lambda, \mu$, and thermoelastic constants $\gamma, \eta$ and $x$. In Cartesian coordinate system, such medium is described by the system of equations $[2,3,8]$ :

Here are the components of the displacement vector, , is the relative temperature, are the components of the mass force, is the power of the heat source, The stress tensor $(x, t)$ is associated with displacements by Duhamel-Neumann law: 
Everywhere there is the summation throughout the repeated indices within the specified limits of variation. Substituting (1.2) into (1.1), we obtain a closed system of equations for which we write in the form:

where the following differential operators are introduced:

This system has mixed hyperbolic-parabolic type. Waves, propagating in a thermoelastic medium, can be shock waves. The equation of the wave front $F$ has the form:

$=0,(1.4)$

where is the main part of the operator, containing only the highest derivatives of the second order, and is a differential operator of motion equations of corresponding elastic medium with parameters $(\lambda, \mu, \rho)$.

We denote by the normal vector to in. It follows from (1.4) that either

or either

Equation (1.5) describes the characteristic surface of the classical parabolic equation, which has the form and does not determine the wave front in space. Equation (1.6) describes wave fronts moving in with the speed:

,,$(1.7)$

where, is the velocity of dilatation waves, is the velocity of shear waves. Consequently, wave fronts (thermoelastic shock waves) in the medium move with the speeds of elastic waves.

In order to continuity conditions of a medium to be preserved and to be a solution (1.1), the following conditions for jumps on characteristic surfaces must be satisfied [7]:

,,$(1.8)$

,, .

Let us introduce the wave vector in, , directed towards the propagation of the shock wave. These equalities imply the conditions on jumps at the shock fronts in :

Here $n$ is the wave vector perpendicular to and has the direction of wave propagation. Equality (1.9) is the condition for conservation the continuity of the medium, (1.10) coincides with the well-known law of conservation of momentum at the fronts of shock waves in elastic media [9].

It follows from (1.9) and (1.11) that the temperature is continuous at the wave fronts, but its gradient suffers a jump proportional to the jump of the normal component to the front of the velocity of the medium displacements.

We will call a solution of equations (1.1), satisfying conditions (1.9) - (1.11) on wave fronts, classical solution.

2. Statement of boundary value problems of uncoupled thermoelasticity. If to set loads at the boundary of a body or mass forces in the body itself, this leads to deformation. At low strain rates in the equation for the temperature field (1.1), the rate of volumetric deformation of the medium $(\eta=0)$ can be neglected. Then the boundary value problem is divided into two tasks: determination of the temperature 
field, after which it becomes possible to determine the field of displacements and stresses in the medium. This model is calleduncoupled thermoelasticity (thermal stress theory).

Consider the following boundary value problems for this model. Let a thermoelastic medium occupies a region bounded by a closed Lyapunov surface $S$ with an external normal $n(x)$. The equations of medium motion in this model are as follows:

Here, is volume force in medium.

Initial conditions (Cauchy conditions ) are given:

,,,,,$(2.2)$

$B V P$ 1. At the boundary (), the acting load and heat flow are known:

,$(2.3)$

where - functions enterable on S.

$B V P$ 2. For given loads and temperature:

,,,$(2.4)$

$B V P$ 3. For the given displacement and temperature:

,,,,$(2.5)$

$B V P$ 4. For given displacements and heat flux:

,,,$(2.6)$

Here $\mathrm{C}(\ldots)$ is the class of continuous functions on the indicated set, $\mathrm{C}$ ' $(\ldots)$ are piecewise continuous bounded functions, $\mathrm{C}^{1}(\ldots)$ are continuously differentiable functions.

At the fronts, the solutions satisfy the jump conditions (1.9)-(1.11). It is required to find displacements, temperature, stresses in the medium.

To solve problems, we use the method of generalized functions (GFM), the main ideas of which are presented in [10-13].

3. Statement of BVP in the space . Let consider equations (2.1) in the space-time of generalized vector functions:

$=$,

where is generalized function, [13].

We introduce the characteristic function of a domain :

We consider the following regular generalized functions:

where $H(t)$ is Heaviside function, are classical solutions of BVP.

Using generalized derivatives of functions: 
where is the singular generalized function - a simple layer on S [13], we get the their partial derivatives:

,

Then Eqs (2.1) in take the form:

Here is the tensor of elastic constants, which for an isotropic medium has the form:

It is seen from formulas (3.2) and (3.3) that the boundary conditions entered in the form of densities of simple and double layers on $\mathrm{S}$, as surface forces and heat sources, and the initial conditions as impulse conditions acting at the moment $t=0$. Note that, when differentiating, we took into account the conditions at the fronts (1.9)-(1.11), which nullify simple and double layers at the fronts of shock waves.

Next, we construct generalized solutions to these boundary value problems for plane deformation ().

4. Determination of the temperature field at plane deformation. To determine the temperature of the medium, we use the Green function of the heat equation $(2.1)_{2}$, corresponding to a pulsed concentrated heat source [12]:

We get the generalized solution (3.3) in the form of a convolution of the Green function with acting sources in $(3.3)$ :

Here we use differentiation properties of a convolution [12]. A variable under the convolution $(*)$ means that it's incomplete convolution, which is taken only for this variable. If there is no such symbol, then this is a complete convolution over $(\mathrm{x}, \mathrm{t})$.

This formula for can be represented in the following integral form:

where is surface differential.

Formula (4.2) allows for the given values of temperature and heat flux at the boundary and the initial temperature to determine the temperature field inside the region under the action of various heat sources.

For $x[?] S, t>0$, the same formula gives a singular BIE for determining the unknown temperature or heat flux at the boundary:

Here, .

The proof of this formula for the boundary points can be carried out in the original space-time. However, it directly follows from the BIE for the Laplace transform of temperature [6].

After solving this equation, the unknown boundary functions of the BVP are determined on the boundary. After that, using formula (4.2), one can determine the temperature in the entire domain .

\section{Generalized solution of equations for displacements and its regularization.}

Taking into account (1.2) Eq (3.2) can be written in the form: 
where is Lamé operator of motion equations of an elastic medium:

To determine the displacements, we use the Green tensor of these equations:

and radiation conditions:

by and by . (5.3)

At plane deformation it is expressed by the formula $[10,14]$ :

It has the following symmetry properties:

Investigation of the asymptotic properties of this tensor showed that it has a weak singularity at wave fronts , does not have singularities for fixed $\mathrm{t}>0, r-0$.

Using the properties of the Green tensor and the differentiation properties of the convolution, we represent the generalized solution (5.1) in the form of a tensor-functional convolution:

Further, it is not possible to write down relation (5.5) in integral form, because the derivatives in the second term are hypersingular at the wave fronts .

To regularize formula (5.5), we introduce the Green tensor antiderivative on $t$ :

The singularities of the tensor were studied in [14]. It is continuous at wave fronts and has a logarithmic singularity at .

Using and the rules for differentiating the convolution, the second term in relation (5.5) can be represented in a regularized form:

As a result, the generalized solution (5.5) takes the regularized form:

which can be written in integral form. To do this, let consider the stress tensor and related tensors.

6. Fundamental stress tensors. Regularization. We introduce the following fundamental stress tensors generated :

Tensor describes stresses on an area with a normal $n$ generated by impulse concen-trated forces at the origin. The tensor is a solution to the Lame equations at and describes the displacement of the medium under the action of a pulsed concentrated source of the multipole type $[14,15]$. It is antisymmetric on $x$ and $n$ :

For integral representation of (5.7), we introduce the antiderivative ont tensor of fundamental stresses $\mathrm{W}(\mathrm{x}, \mathrm{t}, \mathrm{n})$ :

We integrate this tensor at wave fronts and represent it in the form: 
The dynamic tensor has a weak integrable singularity at wave fronts.

The tensor is the fundamental Green stress tensor of static Lame equations. It satisfies the homogeneous Lame equations at and has only one singularity at $x=0$ :

where $K_{i j}$ are bounded.

An analogue of the Gauss formula has been proved for it $[14,15]$ :

. (6.4)

The integral is singular only for boundary points and taken in the sense of the value principal sence.

7. Regular integral representation of displacements. Using the antiderivative fundamental stress tensor (6.2) and their symmetry properties, the solution (5.7) can be written in integral form:

Here, for , all integrals are regular with weak singularities of kernels at wave fronts. With known field temperature and boundary values of displacements and stresses, these formulas make it possible to determine displacements at any point of an elastic medium for any mass forces, including singular ones, which describe the effect of concentrated and impulse power sources of various types. In this case, the component wise convolution should be taken according to the convolution rules in the space of generalized functions.

To solve BVPs, it is necessary to determine the displacements or stresses at S.

8. Resolving singular integral equations. The following theorem is true.

Theorem. Solutions of BVPs 1-4 satisfy the boundary integral equations on $S$ :

for .

Proof. Let, Let consider the second term in the first integral in (7.1), which, taking into account (6.3), can be transformed to the form:

In the integral

we consider the first term on the right for :

The limit of the second integral in (8.2) in the sum with the second integral in (7.1) is reduced to the form:

Taking into account these relations from formula (7.1), passing to the limit to the boundary of the domain, we obtain the formula of the theorem.

For the solution of the first or second boundary value problem, the formulas of the theorem are resolving singular BIEs for determining the displacements. For the third and fourth BVP, the stresses on the boundary are unknown in the formulas of the theorem, i.e. we have a BIEs with a weakly singular kernel:

where all are known, are calculated by the formulas of the theorem.

After determining the unknown boundary functions, using formulas (7.1), you can determine the displacement at any point in the region. After determining the displacements and temperatures using the DuhamelNeumann formulas (1.2), the stresses in the medium are calculated. That solves the BVP.

Conclusion. The constructed boundary integral equations are no classical type. They are very different from BIEs of BVPs problems for elliptic and parabolic equations for which various mathematical methods 
are well developed. In particular, the use of the method of successive approximations is difficult here due to the presence of an unknown velocity of displacements (for the 1st and 2nd BVP). However, the use of numerical methods based on the boundary element method makes it possible to effectively solve this type of equations.

The resulting formulas (4.2) and (7.1) have an important engineering application. They make it possible to determine the thermally stressed state of the medium by the boundary values of stresses, displacements, temperature and heat flux, without solving singular BIEs. Because for real engineering problems these process characteristics can be experimentally measured at the boundary. Moreover, the formulas allow to calculate the influence of each of these characteristics of the process on its stress-strain state. The last one is very important in designing structures made of thermoelastic materials.

This work was supported by grant from the Ministry of Education and Science of the Republic of Kazakhstan ( AP09258948).

\section{REFRENCES}

Biot M.A. Thermoelasticity and irreversible thermodynamics. Journal Apply Physics . 1956; 27(3): 240-253.

2. Novatskiy V. Dynamic problems of thermoelasticity. Moscow, Russia: Mir; 1970, 1- 256.

3. Novatsky V. Theory of elasticity. Moscow, Russia: Mir; 1975, 722- 792 .

4. Danilevskaya V.I. On the dynamic problem of thermoelasticity. Applied Mathematics and Mechanics. 1952; 16(3): 341-344.

5. Hetnarsri R.B. Encyclopedia of thermal stress. Springer (Dordrecht); 2014.

https://doi.org/10.1007/978-94-007-2739-7.

6. Alekseeva L.A., Dadaeva A.N., Zhanbyrbaev N.B. Method of boundary integral equations in boundary value problems of unconnected thermoelastodynamics. Applied Mathematics and Mechanics. 1999; 63(5). 853-859.

7. Alexeyeva L.A., Alipova B.N., Dadayeva A.N. Shock waves as generalized solutions of thermoelastodynamics equations. On the uniqueness of boundary value problems solutions. Journal American Institute of Physics Conference Proceeding. 2017; 1798: 020003-1-020003-8. DOI: 10.1063 / 1.4972595.

8. Kupradze V.D., Gegelia T.G., Basheleishvili M.O., Burchuladze T.V. Three-dimensional problems of the mathematical theory of elasticity and thermoelasticity. Moscow, Russia: Nauka; 1976, 373-418.

9. Petrashen G.I. Wave Propagation in Anisotropic Elastic Media. Moscow, Russia: Nauka; 1980, 157-190.

10. Alekseeva L.A. Analogs of Kirchhoff and Somiliana's formulas in plane problems of elastodynamics. Applied Mathematics and Mechanics. 1991; 55(2): 298-308.

11. Alekseeva L.A. Dynamic analogs of the Green and Gauss formulas for solutions of the wave equation in $\mathrm{R}^{\mathrm{N} *}$. Differential Equations. 1995; 31(11): 1951-1953.

12. Vladimirov V.S. Generalized functions in mathematical physics. Moscow, Russia: Nauka; 1988 , 84- 201.

13. Alexeyeva L. A., Dadayeva A. N., Ainakeyeva N. Zh. Green tensor and regular solutions of equations of rods thermodynamics and their properties. Journal of Theoretical and Applied Mechanics. 2021; 59 (2): 227-238. DOI.org:10.15632/jtam-pl/133233

14. Aitaliev Sh.M., Alexeyeva L.A., Dildabaev Sh.M., Zhanbyrbaev N.B. The method of boundary integral equations in problems of dynamics of elastic multiply connected bodies. Alma-Ata, Kazakhstan: Nauka; $1992,12-83$. 
15. Alexeyeva L.A., Zakiryanova G.K. Generalized solutions of initial-boundary value problems for secondorder hyperbolic systems. Journal of Computational Mathematics and Mathematical Physics. 2011; 51(7): 1280-1293.

16. Alexeyeva L.A., Zakiryanova G.K. Generalized solutions of boundary value problems of the dynamics of deformable solids. Almaty, Kazakhstan: Ashikbayeva; 2020, 109-120. 